\title{
Vingt ans de surveillance du diabète grâce au Système canadien de surveillance des maladies chroniques
}

\author{
Allana G. LeBlanc, Ph. D.; Yong Jun Gao, M. Sc.; Louise McRae, B. Sc.; Catherine Pelletier, M. Sc.
}

Diffuser cet article sur Twitter

\section{Résumé}

En 1999, le gouvernement du Canada, en collaboration avec les provinces et les territoires, met sur pied le Système national de surveillance du diabète (SNSD) afin de suivre l'évolution des taux de diabète au Canada. Le SNSD utilise alors une nouvelle méthode pour recueillir et communiquer les données nationales sur le diabète de façon systématique, au moyen de bases de données administratives sur la santé reliées entre elles. Depuis, il a évolué pour devenir le Système canadien de surveillance des maladies chroniques (SCSMC) et fournit de l'information sur plus de 20 maladies chroniques. Cet article présente les données les plus à jour du SCSMC sur les taux de diabète au Canada. Actuellement, 8,8 \% des Canadiens (9,4 \% d'hommes et 8,1 \% de femmes d'un an et plus) sont atteints de diabète, et environ 549 nouveaux cas sont diagnostiqués chaque jour. Depuis 2000, le taux de prévalence normalisé selon l'âge a augmenté en moyenne de 3,3 \% par année, le taux d'incidence normalisé selon l'âge est demeuré relativement stable et les taux de mortalité toutes causes confondues chez les personnes atteintes de diabète ont diminué en moyenne de 2,1 \% par année. Cela laisse entendre que l'on vit plus longtemps avec un diagnostic de diabète.

\section{Introduction}

Le diabète sucré est un trouble métabolique caractérisé par une déficience de la sécrétion ou de l'action de l'insuline et entraînant une hyperglycémie. Le diabète peut causer un éventail de complications à long terme, en particulier des maladies cardiovasculaires, une rétinopathie, une néphropathie, une neuropathie, des amputations et une diminution de l'espérance de vie ${ }^{1}$. La majorité des cas de diabète relève de deux catégories : diabète de type 1 ou diabète de type 2 . Au sein de la population générale, on estime qu'environ $90 \%$ des diagnostics de diabète correspondent au type 2, $9 \%$ au type 1 et $1 \%$ relèvent d'autres types (p. ex. diabète gestationnel, autres types particuliers liés aux gènes ou à des interactions médicamenteuses) ${ }^{1}$. Le diabète de type 1 est une maladie auto-immune qui ne peut être prévenue ${ }^{1}$. Le diabète de type 2 est dû à un ensemble de facteurs sociaux, environnementaux et génétiques ${ }^{1,2}$.
Les facteurs de risque du diabète de type 2 sont l'obésité, une mauvaise alimentation (p. ex. une alimentation principalement constituée d'aliments ultra-transformés), l'inactivité physique, un faible statut socioéconomique, le vieillissement et l'origine ethnique (p. ex. risque accru chez les Canadiens noirs, les Canadiens sudasiatiques et les peuples autochtones) $)^{2,3}$. Les données de l'Enquête canadienne sur les mesures de la santé indiquent qu'environ $7,3 \%$ des Canadiens (de 12 ans et plus) sont atteints de diabète. Toutefois, ce chiffre est basé sur des données fournies par les répondants, ce qui peut conduire à une sous-estimation ${ }^{4}$.

Pour relever le défi posé par le diabète au Canada, le Gouvernement du Canada, en collaboration avec les provinces et les territoires, a créé le Système national de surveillance du diabète (SNSD) en 19995,6. Plus spécifiquement, le SNSD a été créé pour effectuer une surveillance continue
Points saillants

- Le Système canadien de surveillance des maladies chroniques fournit des renseignements importants sur les taux de diabète au Canada depuis 2000.

- Actuellement, 8,8 \% des Canadiens (9,4 \% des hommes et $8,1 \%$ des femmes d'un an et plus) sont atteints de diabète.

- Le taux de prévalence du diabète normalisé selon l'âge a augmenté avec le temps, tandis que le taux d'incidence normalisé selon l'âge est demeuré stable.

- Le taux de mortalité toutes causes confondues chez les personnes atteintes de diabète a diminué, ce qui donne à penser que l'on vit plus longtemps avec un diagnostic de diabète.

du diabète et de ses complications, pour créer une base de données nationale normalisée en intégrant des bases de données nouvelles et préexistantes, pour diffuser des renseignements comparatifs nationaux visant à orienter l'élaboration de stratégies efficaces en matière de prévention et de traitement du diabète et enfin pour fournir une base à l'évaluation des questions économiques et financières liées aux soins, à la gestion et au traitement du diabète au Canada ${ }^{5}$. Depuis son lancement, le SNSD a évolué pour devenir le Système canadien de surveillance des maladies chroniques (SCSMC), relevant aujourd'hui de l'Agence de la santé publique du Canada (ASPC), et il fournit actuellement des données sur 20 maladies chroniques ${ }^{5}$. Le SCSMC a été 
utilisé pour rendre compte de diverses tendances en lien avec des maladies chroniques au Canada, en particulier l'incidence, la mortalité et la multimorbidité ${ }^{7-10}$. Le SCSMC ayant publié son premier rapport sur le diabète en 2000, 2019 marque 20 ans de surveillance du diabète à l'aide du système. Cet aperçu fournit des données sur les tendances en matière de prévalence, d'incidence et de mortalité toutes causes confondues du diabète au Canada depuis la création du SCSMC.

\section{Méthodologie}

\section{Le Système canadien de surveillance des maladies chroniques}

Grâce au SCSMC, les dossiers qui figurent dans le registre d'assurance-maladie des provinces et des territoires sont jumelés, au moyen d'un identificateur personnel unique, aux demandes de remboursement de médecins, aux dossiers de congé des hôpitaux et aux dossiers des médicaments sur ordonnance correspondants, dans le but de fournir des renseignements sur l'incidence, la prévalence et la mortalité11-13. Les données sur les cas de maladie recensés sont extraites, pour chaque province et territoire, à l'aide d'une approche analytique normalisée reposant sur les définitions de cas relevant de chaque maladie ou affection du SCSMC. Les données individuelles sont regroupées à l'échelle provinciale ou territoriale avant d'être présentées à l'ASPC, dans le but de protéger la vie privée des patients. Elles sont recueillies et mises à jour régulièrement et fournissent de l'information par groupe d'âge, sexe, province/ territoire et tendances au fil du temps. Le SCSMC fournit des données sur tous les Canadiens admissibles à l'assurance-maladie provinciale ou territoriale, soit près de $97 \%$ de la population.

\section{Définition de cas de diabète}

Les Canadiens âgés d'un an ou plus sont considérés comme ayant reçu un diagnostic de diabète s'ils ont au moins un dossier d'hospitalisation ou au moins deux demandes de paiement de médecins en deux ans avec un code de la Classification internationale des maladies (Classification statistique internationale des maladies et des problèmes de santé connexes, ou CIM) correspondant au diabète. La définition de cas actuelle ne permet pas de faire la distinction entre le diabète de type 1 et le diabète de type 2 et rend donc compte du regroupement des deux types. La prévalence correspond à la prévalence à vie et l'incidence correspond à la première fois que le patient a répondu aux critères du diabète. Les codes CIM valides sont : CIM9-MC : 250, et CIM-10-CA : E10, E11, E12, E13, E14. Pour tenir compte du diabète gestationnel, les dossiers contenant des codes pour le diabète sont supprimés chez les femmes de 10 à 54 ans pendant 120 jours avant et 190 jours après l'inscription de tout code lié à une grossesse ou à des services d'obstétrique. Certaines provinces et certains territoires éliminent d'autres renseignements : sont ainsi exclues les données de la Nouvelle-Écosse pour les jeunes de 1 à 19 ans, les données du Yukon avant 2010-2011 et les données du Nunavut avant 2005-2006; ne sont pas disponibles les données de la Saskatchewan pour 2016-2017. Des détails supplémentaires sur l'étude de cas sur le diabète sont disponibles sur l'outil de données en ligne du SCSMC (https://sante-infobase.canada.ca/scsmc /outil-de-donnees/) $)^{3}$ et ailleurs ${ }^{5}$.

\section{Analyse statistique}

Les données du SCSMC présentées dans cet aperçu correspondent à la mise à jour de mars 2019 et portent sur les Canadiens d'un an et plus ayant reçu un diagnostic de diabète de type 1 ou de type 2 et après exclusion du diabète gestationnel. Elles sont présentées pour chaque exercice financier (soit du $1^{\text {er }}$ avril au 31 mars). Les taux bruts reposent sur les effectifs arrondis aléatoirement au multiple de 10 le plus proche. Les taux normalisés selon l'âge reposent sur des effectifs non arrondis et normalisés selon les données postcensitaires finales de la population canadienne de 2011, publiées en 2013, en utilisant des groupes d'âge tout au long de la vie. Le ratio des taux de mortalité a été calculé en divisant le taux de mortalité toutes causes confondues chez les personnes atteintes de diabète par le taux de mortalité toutes causes confondues chez les personnes non diabétiques. Un ratio de taux supérieur à 1 indique que la mortalité est plus élevée chez les personnes atteintes de la maladie que chez les personnes non atteintes, et ce, quelle que soit la cause du décès. Si l'on suppose que les taux de mortalité de référence normalisés selon l'âge des personnes avec la maladie et ceux des personnes sans la maladie sont semblables, on peut attribuer la différence des taux de mortalité toutes causes confondues, représentée par le ratio de ces taux, aux décès directement ou indirectement liés à la maladie. De plus amples renseignements se trouvent en ligne dans le résumé des méthodes du SCSMC (https://sante-infobase .canada.ca/scsmc/outil-de-donnees /Methods).

Nous avons utilisé le logiciel Joinpoint pour exécuter les modèles de régression séquentielle destinés à calculer la variation des taux normalisés selon l'âge au fil du temps et à repérer tout changement statistiquement significatif des tendances entre 2000-2001 et 2016-2017 (logiciel Joinpoint version 4.2.0.2, National Cancer Institute, Bethesda, Maryland, États-Unis). Cela nous a permis de cerner les périodes où la variation annuelle en pourcentage différait considérablement. Le nombre maximal de points de jonction a été fixé à quatre. Le nombre minimal d'observations d'un point de jonction au début ou à la fin des données et le nombre minimal d'observations entre les points de jonction ont été fixés à quatre. Les taux normalisés selon l'âge tiennent compte des différences dans la structure d'âge de la population au fil du temps. Nous avons utilisé la version $9.3 \mathrm{du}$ progiciel SAS (SAS Institute Inc., Cary, Caroline du Nord, États-Unis) pour toutes les autres analyses statistiques. La signification statistique a été fixée à $p<0,05$.

\section{Résultats et analyse}

Les taux de diabète sont présentés dans le tableau 1. En 2016-2017, environ 8,8 \% des Canadiens $(9,4 \%$ chez les hommes et $8,1 \%$ chez les femmes; population d'un an et plus) étaient atteints de diabète. La prévalence du diabète était plus élevée chez les adultes que chez les enfants et les jeunes (10,9\% contre 0,3\%). Cela signifie qu'en 2016-2017, environ 3,2 millions de Canadiens vivaient avec le diabète (figure 1-A), soit environ 1 adulte sur 11 (20 ans et plus) et 1 enfant ou 1 jeune sur 333 (1 à 19 ans). Depuis 2000-2001, les taux de prévalence normalisés selon l'âge ont augmenté en moyenne de 3,3\% par année ( $p<0,001$; figure 1-A). La plus forte augmentation, 5,3 \% en moyenne, a eu lieu entre 2000-2001 et 2006-2007 ( $p<0,001$ ). Entre 2006-2007 et 2010-2011, le taux de prévalence normalisé selon l'âge a augmenté en moyenne de 3,3 \% par année ( $p$ < 0,001); de 2010-2011 à 2016-2017, il a augmenté en moyenne de 1,2\% par année $(p<0,001)$.

En 2016-2017, le taux de nouveaux cas de diabète diagnostiqués était de 603,5 pour 
TABLEAU 1

Taux de diabète au Canada, Système canadien de surveillance des maladies chroniques, 2016-2017

\begin{tabular}{|c|c|c|c|}
\hline Indicateur & Total & Hommes & Femmes \\
\hline \multicolumn{4}{|l|}{ Prévalence (n, \%) } \\
\hline Population totale ( 1 an et plus) & $3170969(8,8)$ & $1686700(9,4)$ & $1484260(8,1)$ \\
\hline Enfants et jeunes (1 à 19 ans) & $24330(0,3)$ & $12700(0,3)$ & $11630(0,3)$ \\
\hline Adultes (20 ans et plus) & $3146630(10,9)$ & $1674010(11,8)$ & $1472630(10,0)$ \\
\hline \multicolumn{4}{|l|}{ Incidence (n, taux pour 100 000) } \\
\hline Population totale (1 an et plus) & $200400(603,5)$ & $109230(667,9)$ & $91160(540,9)$ \\
\hline Enfants et jeunes (1 à 19 ans)) & $3070(42,1)$ & $1530(40,9)$ & $1540(43,3)$ \\
\hline Adultes (20 ans et plus) & $197330(761,5)$ & $107700(853,7)$ & $89630(674,0)$ \\
\hline $\begin{array}{l}\text { Taux de mortalité (population } \\
\text { totale d' } 1 \text { an et plus; IC à } 95 \% \text { ) }\end{array}$ & 1,96 (1,91 à 2,02) & $1,80(1,74$ à 1,85$)$ & $2,11(2,00$ à 2,23$)$ \\
\hline
\end{tabular}

Abréviation : IC, intervalle de confiance.

Remarque : Les données, à jour en date de mars 2019, comprennent les diabètes de type 1 et de type 2 diagnostiqués combinés mais excluent le diabète gestationnel. Sont exclues les données de la Nouvelle-Écosse pour les 1 à 19 ans, les données du Yukon avant 2010-2011 et les données du Nunavut avant 2005-2006; ne sont pas disponibles les données de la Saskatchewan pour 2016-2017.

100000 Canadiens (d'un an et plus). Cela représente environ 200400 nouveaux cas par année, soit 549 nouveaux cas par jour (figure 1-B). Entre 2000-2001 et 2006-2007, le taux d'incidence normalisé selon l'âge a augmenté en moyenne de 2,2\% par année $(p<0,001)$ et, entre 2006-2007 et 20162017, il a diminué en moyenne de 2,2 \% par année $(p<0,001)$. Cela signifie que, tout au long de la période de surveillance, les taux d'incidence normalisés selon l'âge sont demeurés relativement stables (variation annuelle moyenne en pourcentage : $-0,5, p=0,10)$. En 2016-2017, le taux de mortalité toutes causes confondues chez les personnes atteintes de diabète était de 1020,6 pour 100000 , contre 519,5 pour 100000 chez les personnes non diabétiques (figure 1-C). Entre 2000-2001 et 2016-2017, le taux de mortalité toutes causes confondues a diminué (variation annuelle moyenne en pourcentage : $-2,1, p<0,001)$. Cette tendance a été particulièrement prononcée entre 2000-2001 et 2013-2014, avec une baisse du taux de mortalité toutes causes confondues de $2,9 \%(p<0,001)$. Entre 2013-2014 et 2016-2017, il n'y a eu aucun changement (variation annuelle en pourcentage : $1,7 \%, p=0,30)$. Comparativement aux personnes qui ne sont pas atteintes de diabète, le taux de mortalité des personnes qui en sont atteintes est environ 1,96 fois plus élevé (intervalle de confiance [IC] à $95 \%: 1,91$ à 2,02).

\section{Points forts et limites}

Comparativement à d'autres systèmes ou enquêtes de surveillance, le SCSMC offre plusieurs avantages. Il permet de recueillir des données sur l'incidence (plus sensibles aux variations épidémiologiques de la maladie qu'à la prévalence), d'examiner les tendances au fil du temps, de produire des données comparables entre les provinces et les territoires (aller sur la page https:// sante-infobase.canada.ca/scsmc/outil-de -donnees/ et sélectionner " diabète » et " comparaisons géographiques ») et d'inclure des données à l'échelle de la population fondées sur les maladies ou affections diagnostiquées sur le plan médical. La principale lacune de cette étude réside dans le fait que la définition de cas actuelle ne permet pas de faire la différence entre le diabète de type 1 et le diabète de type 2 . Le SCSMC se limite également au diabète diagnostiqué chez les utilisateurs des services de santé. Cela peut conduire à une sous-estimation de la prévalence, due au diabète subclinique ou non diagnostiqué. L'influence d'autres facteurs contextuels susceptibles d'infléchir l'évolution des taux (p. ex. l'origine des différences dans les variations annuelles en pourcentage) n'a pas été étudiée. De futures recherches auraient à peaufiner la définition de cas de diabète et notamment à envisager la possibilité d'inclure des bases de données sur les médicaments sur ordonnance $^{14}$. Il serait également utile d'examiner les facteurs contextuels susceptibles d'influencer les taux de diabète au Canada. De futures recherches pourraient également examiner la multimorbidité et calculer les coûts associés à diverses maladies.

\section{Conclusion}

Le SCSMC et son architecture originale fournissent d'excellentes données sur les maladies et les affections chroniques au Canada. Depuis 20 ans, l'ASPC, en partenariat avec l'ensemble des provinces et des territoires, rend compte des taux de diabète à l'échelle nationale. Notre étude fournit les renseignements les plus à jour sur la prévalence, l'incidence et la mortalité du diabète au Canada. En 2016-2017, 8,8 \% des Canadiens étaient atteints de diabète, soit environ 1 adulte sur 11 et 1 enfant ou jeune sur 333. Environ 549 nouveaux cas sont diagnostiqués chaque jour. Le taux de prévalence du diabète normalisé selon l'âge a augmenté avec le temps, tandis que le taux d'incidence normalisé selon l'âge est demeuré relativement stable. Cela peut s'expliquer en partie par le fait que l'on vit plus longtemps avec la maladie, comme en témoigne la baisse du taux de mortalité au fil du temps. Toutefois, le risque de mortalité est beaucoup plus élevé chez les personnes atteintes de diabète que chez celles qui n'en sont pas atteintes, et le diabète demeure l'une des principales maladies chroniques au Canada.

\section{Remerciements}

Ces données ont pu être recueillies grâce à la collaboration entre l'ASPC et les gouvernements provinciaux et territoriaux de la Colombie-Britannique, de l'Alberta, de la Saskatchewan, du Manitoba, de l'Ontario, du Québec, du Nouveau-Brunswick, de la Nouvelle-Écosse, de l'Île-du-Prince-Édouard, de Terre-Neuve-et-Labrador, des Territoires du Nord-Ouest, du Yukon et du Nunavut. Il ne faut pas inférer qu'ils ont été sanctionnés par les provinces et les territoires. Les données provinciales et territoriales ont été envoyées au SCSMC en mars 2019 (données jusqu'en 2016-2017).

\section{Conflits d'intérêts}

Les auteurs déclarent n'avoir aucun conflit d'intérêts.

\section{Contributions des auteurs et avis}

Allana G. LeBlanc a conçu le manuscrit et en a rédigé la première ébauche. Allana G. LeBlanc et Yong Jun Gao ont réalisé les analyses statistiques. Tous les auteurs ont procédé à un examen critique et ont formulé des commentaires sur tous les aspects 
FIGURE 1

Tendances des taux de diabète de 2000-2001 à 2016-2017

\section{A. Nombre total de cas et taux de prévalence du diabète normalisé selon l'âgé}

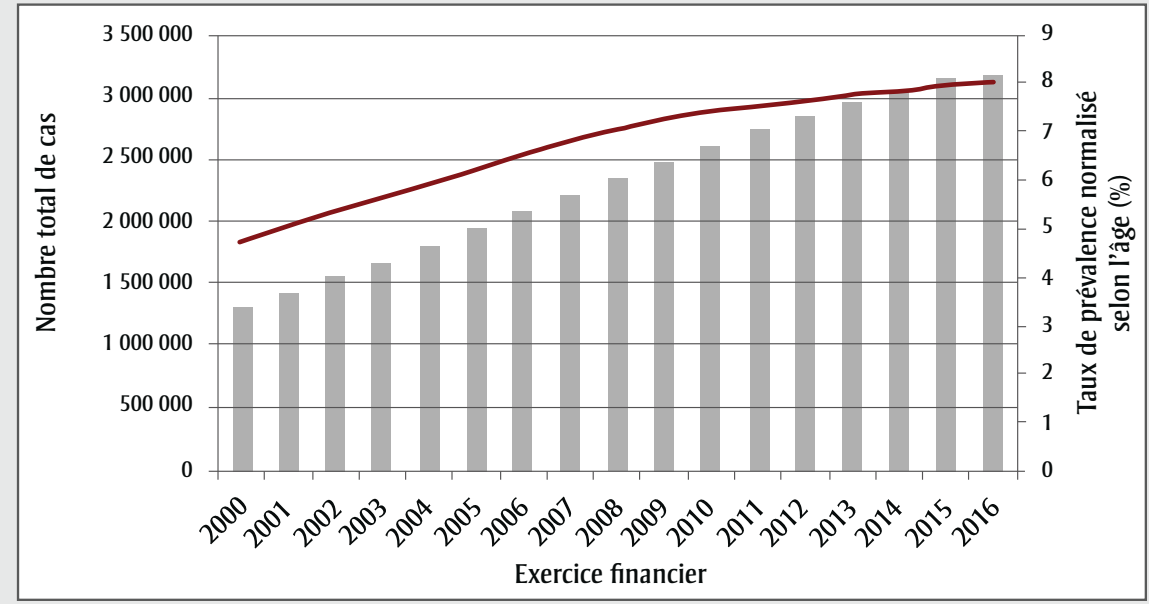

B. Nombre de nouveaux cas et taux d'incidence du diabète normalisé selon l'âge

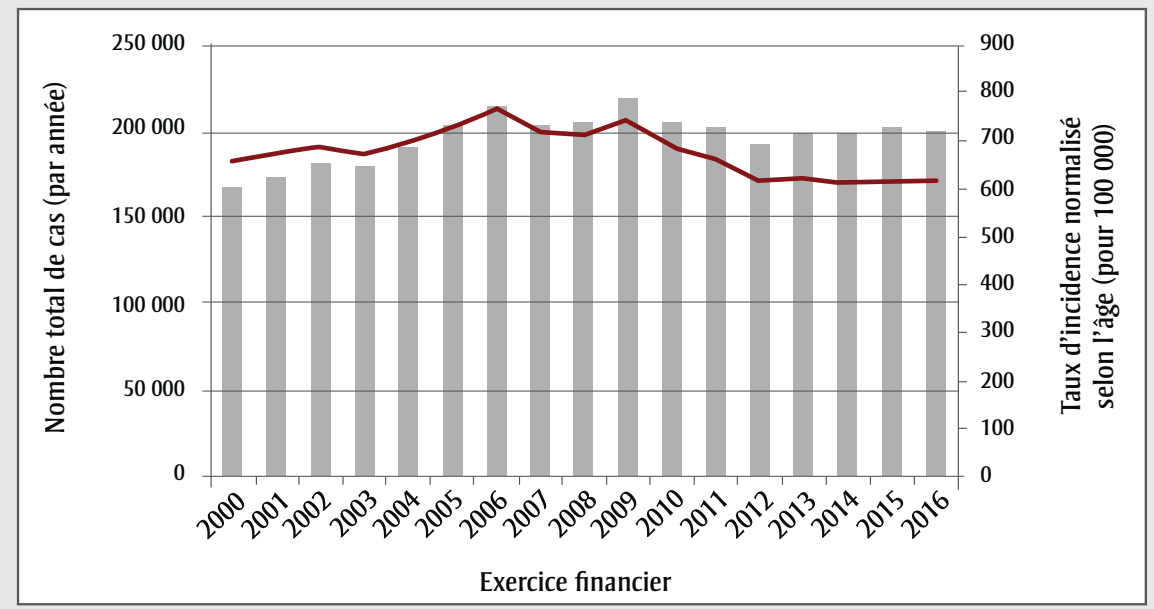

C. Taux de mortalité et ratio des taux de mortalité des personnes atteintes de diabète par rapport à ceux des personnes non atteintes de diabète

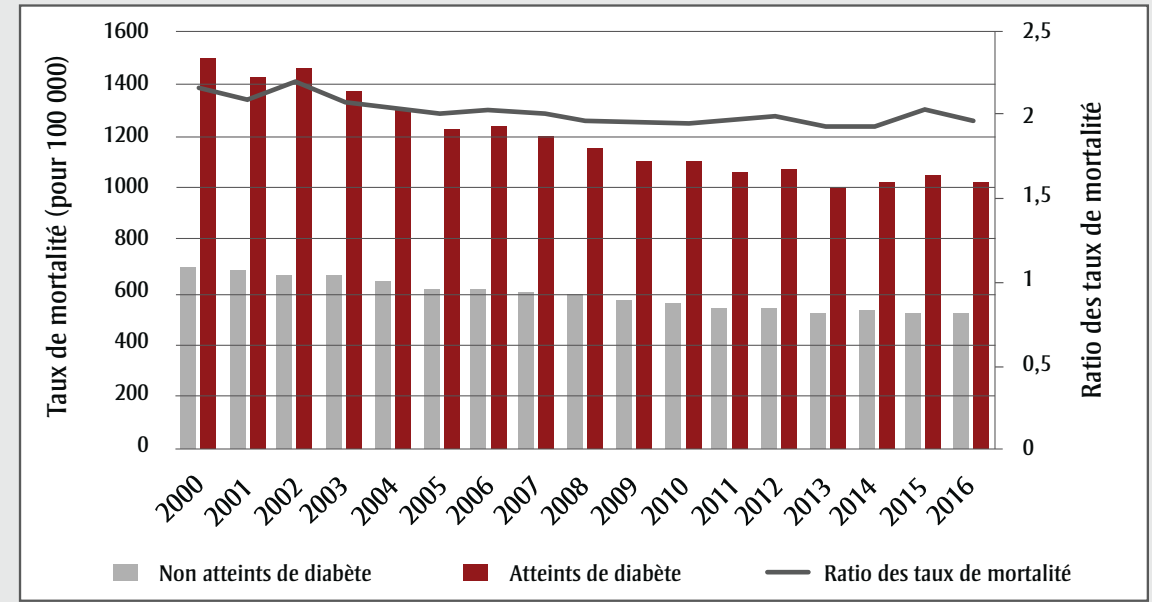

Source des données : Système canadien de surveillance des maladies chroniques (SNSMC).

Remarques : Les données, à jour en date de mars 2019, comprennent les diabètes de type 1 et de type 2 diagnostiqués combinés mais excluent le diabète gestationnel. Sont exclues les données de la Nouvelle-Écosse pour les 1 à 19 ans, les données du Yukon avant 2010-2011 et les données du Nunavut avant 2005-2006; ne sont pas disponibles les données de la Saskatchewan pour 2016-2017. de l'article. Tous les auteurs ont approuvé la version finale de cet aperçu.

Le contenu de l'article et les points de vue qui y sont exprimés n'engagent que les auteurs; ils ne correspondent pas nécessairement à ceux du gouvernement du Canada.

\section{Références}

1. Comité d'experts sur les Lignes directrices de pratique clinique de Diabète Canada. Guide de pratique clinique de Diabète Canada 2018 pour la prévention et la prise en charge du diabète au Canada. Can J Diabète. 2018; 42(Suppl 1):S1-S325.

2. Agence de la santé publique du Canada (ASPC). Les principales inégalités en santé au Canada : un portrait national. Ottawa (Ont.) : ASPC; 2018. En ligne à : https://www.canada.ca/content/dam /phac-aspc/documents/services /publications/science-research/key -health-inequalities-canada-national -portrait-executive-summary/key_ health_inequalities_full_report-fra.pdf

3. Agence de la santé publique du Canada (ASPC). Infobase de la santé publique : Système canadien de surveillance des maladies chroniques (SCSMC) [Internet]. [Sélectionner "diabète" dans le menu "affections »]. Ottawa (Ont.) : ASPC [mis à jour le 14 mars 2019; cité le 17 juillet 2018]. En ligne à: https:// sante-infobase.canada.ca/scsmc/outil -de-donnees/

4. Statistique Canada. Feuillets d'information de la santé - Diabète, 2017. Ottawa (Ont.) : Statistique Canada; 2018 [ $\mathrm{n}^{\circ}$ de catalogue 82-625X].

5. Santé Canada. Relever le défi posé par le diabète au Canada - premier rapport du système national de surveillance du diabète (SNSD). Ottawa (Ont.) : Gouvernement du Canada; 2003 [n de catalogue H39-4/21-2003F].

6. Agence de la santé publique du Canada (ASPC). Le Système canadien de surveillance des maladies chroniques Un aperçu. Ottawa (Ont.) : ASPC [mise à jour le 12 octobre 2018; cité le 10 juillet 2019]. En ligne à : https:// www.canada.ca/fr/sante-publique /services/publications/systeme-canadien -surveillance-maladies-chroniques -fiche-information.html 
7. Comité directeur des IMCC, Agence de la santé publique du Canada. Quel est l'état de santé des Canadiens? Brève mise à jour. Promotion de la santé et prévention des maladies chroniques au Canada. 2018;38(10):434-436. Erratum dans Promotion de la santé et prévention des maladies chroniques au Canada. 2019;39(2):67.

8. Hamm NC, Pelletier L, Ellison J, et al. Tendances des taux d'incidence des maladies chroniques d'après le Système canadien de surveillance des maladies chroniques. Promotion de la santé et prévention des maladies chroniques au Canada. 2019;39(6/7):238-247.

9. Agence de la santé publique du Canada (ASPC). Quel est l'état de santé des Canadiens? Ottawa (Ont,) : ASPC; 2016 [ $\mathrm{n}^{\circ}$ de catalogue : HP40$167 / 2016 \mathrm{~F}]$.

10. Feely A, Lix L, Reimer K. Estimation de la prévalence de la multimorbidité au moyen du Système canadien de surveillance des maladies chroniques. Promotion de la santé et prévention des maladies chroniques au Canada. 2017;37(7):235-243.

11. Lix L, Ayles J, Bartholomew S, et collab. The Canadian Chronic Disease Surveillance System: a model for collaborative surveillance. Int J Pop Data Sci [Internet]. 2018;3(3). En ligne à: https://ijpds.org/article/view/433

12. Lix L, Reimer K. The Canadian Chronic Disease Surveillance System: a distributed surveillance model. Online J Public Health Inform [Internet]. 2017;9(1):e140. En ligne à: https:// doi.org/10.5210/ojphi.v9i1.7726

13. James RC, Blanchard JF, Campbell D, et al. Un modèle de surveillance des maladies non transmissibles au Canada : le Système pilote de surveillance du diabète des Prairies. Maladies chroniques au Canada. 2004;25(1):7-12.

14. Liscombe LL, Hwee J, Webster L, et collab. Identifying diabetes cases from administrative data: a population-based validation study. BMC Health Service Research [Internet]. 2018;18(1):316. 